\title{
Coherent transport on Apollonian networks and continuous-time quantum walks
}

\author{
Xin-Ping $\mathrm{Xu}^{1,3}$, Wei $\mathrm{Li}^{1,2}$, and Feng $\mathrm{Liu}^{1}$ \\ ${ }^{1}$ Institute of Particle Physics, HuaZhong Normal University, Wuhan 430079, China \\ ${ }^{2}$ Max-Planck-Institute for Mathematics in the Sciences, Inselstr. 22, Leipzig, Germany \\ ${ }^{3}$ Institute of High Energy Physics, Chinese Academy of Science, Beijing 100049, China
}

\begin{abstract}
We study the coherent exciton transport on Apollonian networks generated by simple iterative rules. The coherent exciton dynamics is modeled by continuous-time quantum walks and we calculate the transition probabilities between two nodes of the networks. We find that the transport depends on the initial nodes of the excitation. For networks less than the second generation the coherent transport shows perfect revivals when the initial excitation starts at the central node. For networks of higher generation, the transport only shows partial revivals. Moreover, we find that the excitation is most likely to be found at the initial nodes while the coherent transport to other nodes has a very low probability. In the long time limit, the transition probabilities show characteristic patterns with identical values of limiting probabilities. Finally, the dynamics of quantum transport are compared with the classical transport modeled by continuous-time random walks.
\end{abstract}

PACS numbers: 05.60.Gg, 05.60.Cd, 71.35.-y, 89.75.Hc, 89.75.-k

The problem of coherent exciton transport modeled by quantum walks is widely studied and relevant to many distinct fields, such as polymer physics, solid state physics, biological physics and quantum computation [1, 2, 3]. Such studies have been done in the framework of continuous-time quantum walks (CTQWs) and on various discrete systems [4, 5]. It has been shown that the dynamics of coherent transport are strongly influenced by the structure of the underlying discrete systems [6, 7, 8]. Most of previous studies focus CTQWs on graphs with simple structures 9, 10, 11], coherent dynamics on general graphs have not received much attention in the scientific community. To this end, it is natural to consider quantum transport on graphs with general structure embedded in nature.

An important and universal feature of networked systems (or graphs) in nature is that they have the smallworld and scale-free property [12, 13]. The Apollonian networks (ANs) [14, 15] are a very useful toy model that captures all these features simultaneously, thus provide a good facility to study the dynamical processes taking place on networked systems, including percolation, electrical conduction, etc [14, 16, 17].

In this paper, we consider coherent exciton transport on 2D Apollonian networks (ANs). The network can be generated as follows [14]: At the initial generation $g=0$, the network is composed of three fully connected nodes marked as 1, 2, and 3. At the subsequent generation, a new node is added inside each (newly established) triangle and linked to the three vertices of the triangle. Using this simple rule, we can obtain a deterministic 2D ANs of size $N=3+\left(3^{G}-1\right) / 2(G$ is the number of generation) 14]. Many topological properties of this network model have been well-studied in the literature [15, 18]. Fig. 1 shows the structure of an $\mathrm{AN}$ in four generations $(G=4)$.

The coherent exciton transport on a connected net-

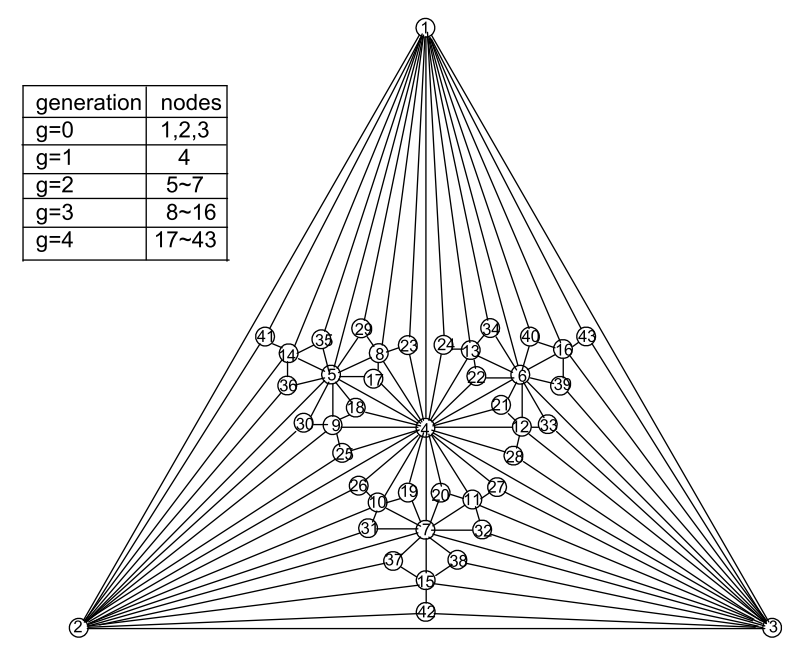

FIG. 1: Apollonian network generated by simple iterative rules in four generations $(G=4)$. The nodes at each generation $g$ are marked as consecutive numbers.

work is modeled by the continuous-time quantum walks (CTQWs), which is obtained by replacing the Hamiltonian of the system by the classical transfer matrix, i.e., $H=-T[19,[20]$. The transfer matrix $T$ relates to the Laplace matrix by $T=-\gamma A$, where for simplicity we assume the transmission rates $\gamma$ of all bonds to be equal and set $\gamma \equiv 1$ in the following [19, 20]. The Laplace matrix $A$ has nondiagonal elements $A_{i j}$ equal to -1 if nodes $i$ and $j$ are connected and 0 otherwise. The diagonal elements $A_{i i}$ equal to degree of node $i$, i.e., $A_{i i}=k_{i}$. The states $|j\rangle$ endowed with the node $j$ of the network form a complete, ortho-normalised basis set, which span the whole accessible Hilbert space. The time evolution of a state $|j\rangle$ starting at time $t_{0}$ is given by $|j, t\rangle=U\left(t, t_{0}\right)|j\rangle$, where $U\left(t, t_{0}\right)=\exp \left[-i H\left(t-t_{0}\right)\right]$ is the quantum me- 

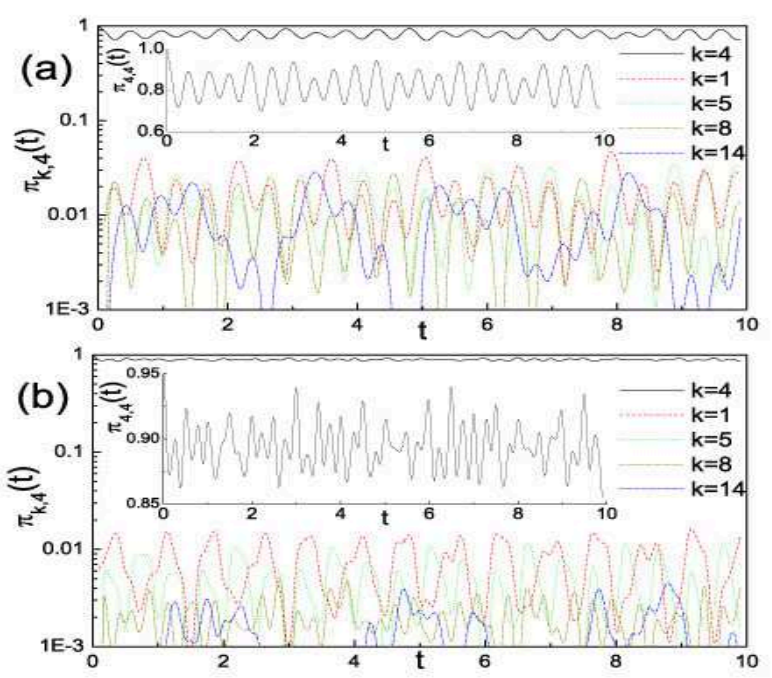

FIG. 2: (Color online) Time evolution of transition probabilities $\pi_{k, 4}(t)$ for different values of $k$ (marked as different types of curves) on ANs of $G=3$ (a) and $G=4$ (b). The excitation starts at central node 4 . The insets are enlarged linear-scale plots of return probability $\pi_{4,4}(t)$.

chanical time evolution operator. The transition amplitude $\alpha_{k, j}(t)$ from state $|j\rangle$ at time 0 to state $|k\rangle$ at time $t$ reads $\alpha_{k, j}(t)=\langle k|U(t, 0)| j\rangle$ and obeys Schrödingers equation [21]. Then the classical and quantum transition probabilities to go from the state $|j\rangle$ at time 0 to the state $|k\rangle$ at time $t$ are given by $p_{k, j}(t)=\left\langle k\left|e^{-t A}\right| j\right\rangle$ and $\pi_{k, j}(t)=\left|\alpha_{k, j}(t)\right|^{2}=\left|\left\langle k\left|e^{-i t H}\right| j\right\rangle\right|^{2}$ [19], respectively. Using $E_{n}$ and $\left|q_{n}\right\rangle$ to represent the $n$th eigenvalue and eigenvector of $H$, the classical and quantum transition probabilities between two nodes can be written as [19, 20, 21]

$$
\begin{gathered}
p_{k, j}(t)=\sum_{n} e^{-t E_{n}}\left\langle k \mid q_{n}\right\rangle\left\langle q_{n} \mid j\right\rangle, \\
\pi_{k, j}(t)=\left|\alpha_{k, j}(t)\right|^{2}=\left|\sum_{n} e^{-i t E_{n}}\left\langle k \mid q_{n}\right\rangle\left\langle q_{n} \mid j\right\rangle\right|^{2} .
\end{gathered}
$$

Generally, to get $p_{k, j}(t)$ and $\pi_{k, j}(t)$, all the eigenvalues $E_{n}$ and eigenvectors $\left|q_{n}\right\rangle$ are required. In the following we will consider $p_{k, j}(t)$ and $\pi_{k, j}(t)$ obtained from diagonalizing the Hamiltonian $H$ by using the standard software package Mathematica 5.0.

We start our analysis by considering transport dynamics on ANs of $G=3(N=16)$ and $G=4(N=43)$ when the excitation starts at the central node 4 . The nodes are numbered according to Fig. 1 and network of $G=3$ (nodes labeled as $1 \sim 16$ ) is a subgraph of $G=4$ (nodes labeled as $1 \sim 43$ ). Due to rotational symmetry, the transition probabilities from node 4 to certain groups of
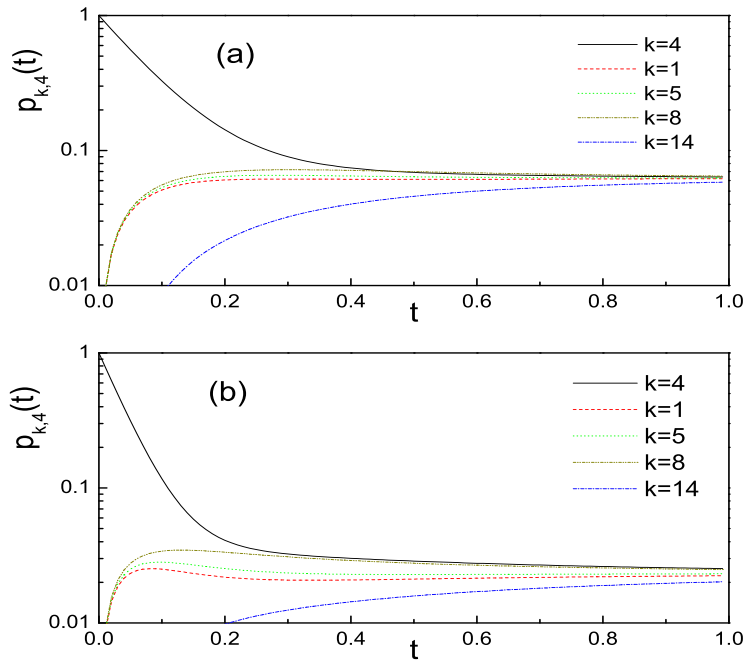

FIG. 3: (Color online) Time evolution of the classical probabilities $p_{k, 4}(t)$ for different values of $k$ (marked as different types of curves) on ANs of $G=3$ (a) and $G=4$ (b). The excitation starts at central node 4 . The classical $p_{k, 4}(t)$ approach the equip-partitioned probability $1 / N$ at long time scale.

nodes are equal. Thus, we choose several different transition probabilities, namely, $\pi_{4,4}(t), \pi_{1,4}(t), \pi_{5,4}(t), \pi_{8,4}(t)$ and $\pi_{14,4}(t)$ for further study.

Fig. 2 shows these quantum transition probabilities for ANs of $G=3$ and $G=4$. We find that there is a high probability to find the excitation at the initial node $\left(\pi_{4,4}(t)\right.$ marked as solid curves in the Fig. 2). To see the behavior of $\pi_{4,4}(t)$ clearly, we display $\pi_{4,4}(t)$ in an enlarged linear scale (See inserted plots in Fig. 2). For AN of $G=3, \pi_{4,4}(t)$ shows regular oscillations, as generation increases, $\pi_{4,4}(t)$ becomes irregular and its average value increases (Compare the inserted plots in Fig. 2 (a) and (b)). Transition probabilities between the initial node and other nodes are considerably low compared to the return probability $\pi_{4,4}(t)$ (See the dashed curves in (a) and (b)). The corresponding transition probabilities $\pi_{k, 4}(t)(k \neq 4)$ of a $G=3 \mathrm{AN}$ is higher than those of a $G=4 \mathrm{AN}$. This may be attributed to the fact that the return probability $\pi_{4,4}(t)$ on a $G=4 \mathrm{AN}$ is larger than that on a $G=3 \mathrm{AN}$.

Fig. 3 shows the classical transition probabilities $p_{k, 4}(t)$ for different values of $k$. It is found that the classical transition probabilities approach the equipartition $1 / N$ very quickly and $p_{14,4}(t)$ reaches $1 / N$ much slower than other transition probabilities. This can be explained by the shortest path length from the initial excitation node 4 . The number of bonds between node 4 and 14 is larger than the distance between other pairs of nodes. In addition, because the shortest path lengths between 4 and 1, 4 and 5, 4 and 8 are equal, the classical 

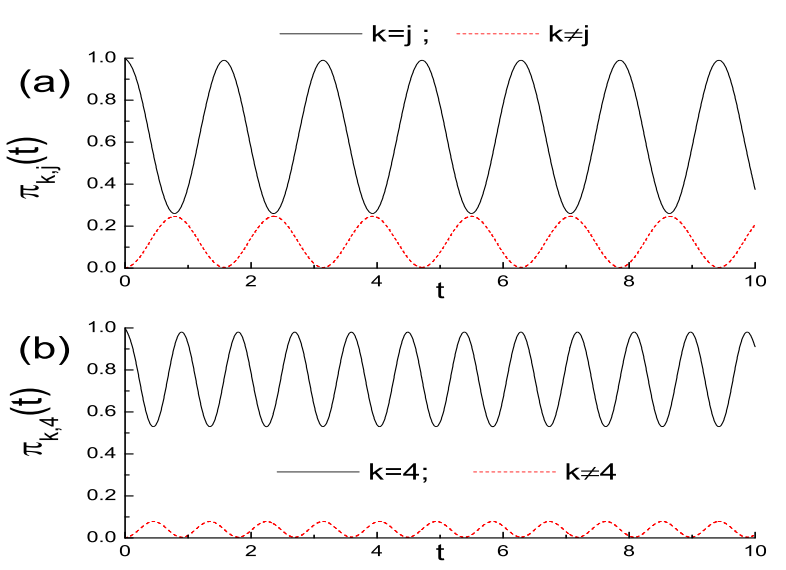

FIG. 4: (Color online) (a) Transition probabilities $\pi_{k, j}(t)$ for AN of $G=1$. (b) Transition probabilities $\pi_{k, 4}(t)$ for AN of $G=2$. Both results are numerically obtained by diagonalizing the Hamiltonian $H$ and consistent with the analytical results in Eqs. (3) and (4).

$p_{1,4}(t), p_{5,4}(t)$ and $p_{8,4}(t)$ are comparable (Compare the curves in Fig. 31). Noting that the long time averaged $\pi_{4,4}(t)$ is much higher than equip-partitioned probability $1 / N$ and other (long time averaged) quantum transition probabilities is less than $1 / N$, we conclude that the classical transport to other nodes (non-initial node) of the network is more efficient than quantum transport.

Interestingly, for $G=1(N=4)$ and $G=2(N=7)$ ANs, the quantum transition probabilities are fully periodic when the coherent excitation starts from the central node 4 . In this case we obtain, based on the analytically determined eigenvalues and eigenvectors 22], that for $G=1, \pi_{k, j}(t)$ have the following, periodic form:

$$
\pi_{k, j}(t)= \begin{cases}(5+3 \cos 4 t) / 8, & k=j, \\ (1-\cos 4 t) / 8, & k \neq j .\end{cases}
$$

And for $G=2$ we have,

$$
\pi_{k, 4}(t)= \begin{cases}(37+12 \cos 7 t) / 49, & k=4, \\ (2-2 \cos 7 t) / 49, & k \neq 4 .\end{cases}
$$

Fig. 4 shows the behavior of $\pi_{k, j}(t)$ obtained by numerically diagonalizing the Hamiltonian $H$ for $G=1$ and $G=2$ ANs. This agrees the analytical results in Eqs. (3) and (4). We find that there is a perfect revival of the initial state for each $t=2 n \pi / N(n \in$ Integers), where $N$ is the number of nodes of the considered network. This revival of the initial probability distribution resembles the results obtained for continuous and discrete quantum carpets [7, 23], in which the revival is only perfect for small size of cycles 7,24 . The case for ANs is analogous: The revivals are perfect for small ANs of $G \leqslant 2$, when the network size becomes larger $(G \geqslant 3)$, there are only partial revivals of the initial state (Compare Fig. 2 and Fig. (4).
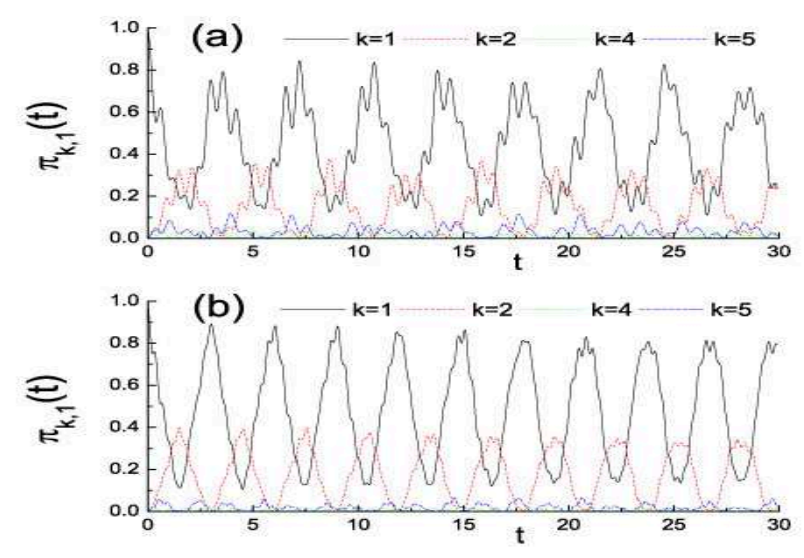

FIG. 5: (Color online) Time evolution of transition probabilities $\pi_{k, 1}(t)$ for different values of $k$ (marked as different types of curves) on ANs of $G=3$ (a) and $G=4$ (b). The excitation starts at noncentral node 1 . The return probability $\pi_{1,1}(t)$ is nearly periodic for both the networks.

Now we turn to the case when the initial excitation starts at other positions of the network. Fig. 5 shows the transition probabilities when the initial excitation is placed at node 1 . For both the $G=3$ and $G=4$ ANs, the return probabilities $\pi_{1,1}(t)$ display regular oscillations. The return probability $\pi_{1,1}(t)$ is much larger than other transition probabilities $\pi_{k, 1}(t)(k \neq 1)$ at most time intervals. It is interesting to note that except for the high return probability $\pi_{1,1}(t)$, there is also considerable transport to nodes 2 and 3 (Note $\pi_{2,1}(t)=\pi_{3,1}(t)$ because of axis-symmetry). Nevertheless, transport to other nodes (such as 4, 5 etc) is particularly low. This suggests that the excitation is preferably located on the nodes of the same generation of the initial node.

If the initial excitation starts from other noncentral nodes, the results are similar but some details change. The oscillation amplitude and period are different and there is also a relative high probability to find the excitation at the initial node.

In order to discuss what happens at long times, we consider the long time averages of the transition probabilities $p_{j, k}(t)$ and $\pi_{j, k}(t)$. On finite ANs, the transition probability converges to a certain value, this value is determined by the long time average. Classically, the long time averaged transition probabilities equal to the equal-partitioned probability $1 / N$. However, the quantum transport does not lead to equipartition. The long time average of $\pi_{j, k}(t)$ is defined as

$$
\begin{aligned}
\chi_{k, j} & =\lim _{T \rightarrow \infty} \frac{1}{T} \int_{0}^{T} \pi_{k, j}(t) d t \\
& =\sum_{n, l} \delta\left(E_{n}-E_{l}\right)\left\langle k \mid q_{n}\right\rangle\left\langle q_{n} \mid j\right\rangle\left\langle j \mid q_{l}\right\rangle\left\langle q_{l} \mid k\right\rangle,
\end{aligned}
$$

where $\delta\left(E_{n}-E_{l}\right)=1$ for $E_{n}=E_{l}$ and $\delta\left(E_{n}-E_{l}\right)=0$ else. Some eigenvalues of $H$ may be degenerate, so the sum in the equation contain terms belonging to differ- 

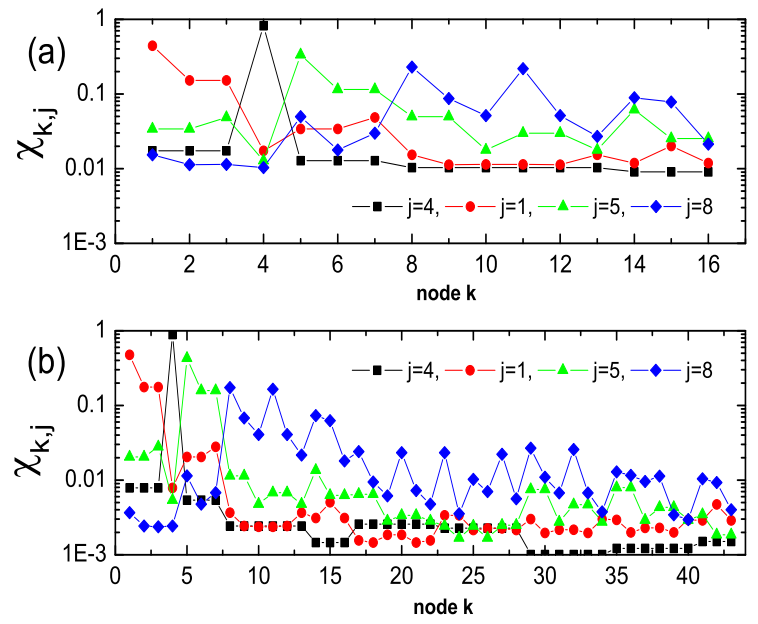

FIG. 6: (Color online) Long time limiting probabilities $\chi_{k, j}$ for different node $j$ of initial excitation on the $G=3$ (a) and $G=4$ (b) ANs. The squares, dots, triangles and rhombus denote initial excitation at node $4,1,5$ and 8 respectively.

ent eigenstates. Here, we consider the limiting transition probabilities $\chi_{k, j}$ according to this equation.

Fig. 6 (a) and (b) show the limiting probability distributions for $G=3$ and $G=4$ ANs. In the figure, we find that $\chi_{j, j}$ is larger than other transition probabilities $\chi_{k, j}(k \neq j)$. This indicates the excitation is most likely to be found at the initial node, which is accord with the observation in Figs. 2 and 5 .

An interesting feature related to the limiting probabilities is that different nodes, $k$ and $l$, may have the same transition probabilities, i.e., $\chi_{k, j}=\chi_{l, j}$. Concretely, for an excitation starting from the central node $j=4$, transport to nodes of certain cluster has identical limiting transition probabilities (See the black squares in Fig. (6). For instance, $\chi_{1,4}, \chi_{2,4}$ and $\chi_{3,4}$ are equal to each other; $\chi_{5,4}=\chi_{6,4}=\chi_{7,4} ; \chi_{k, 4}$ are equal for $8 \leqslant k \leqslant 13$; ... . The nodes of clusters having the same transition probabilities in such case are easy to be identified due to rotation-symmetry of the central node. Furthermore, nodes of the same generation or having the same connectivity may have different limiting probabilities (compare the values of $\chi_{k, 4}$ for cluster $8 \leqslant k \leqslant 13$ and cluster $14 \leqslant k \leqslant 16$ in the Fig. 6).

For an excitation starting from noncentral node, the situation is quite different. When the excitation starts at node 1 (See the dots in Fig 6), $\chi_{2,1}$ equals to $\chi_{3,1}$. Such identical values of transition probabilities are also easy to be distinguished and can be understood as a result of the axis-symmetry. The case for excitation starting at node 5 is analogous (See the triangles in the plots). Particularly, if the excitation starts at node 8 , nodes 10 and
12 have the same limiting probability, i.e., $\chi_{10,8}=\chi_{12,8}$ (rhombus indicated in Fig. 6). Such kind of identical probability is not straightforward to be realized but also can be ascribed to the rotation symmetry of the structure of ANs. If triangle $\Delta 145$ is rotated $\pi / 3$ and $2 \pi / 3$, the initial node 8 changes to the positions 12 and 10 respectively. Except for the equal value of $\chi_{10,8}$ and $\chi_{12,8}$ on both the $G=3$ and $G=4$ ANs, we find that there are more identical probabilities on the $G=4$ AN. For instance, we find the following equal transition probabilities: $\chi_{31,8}=\chi_{33,8}, \chi_{19,17}=\chi_{21,17}, \chi_{26,23}=\chi_{28,23}$, $\chi_{10,29}=\chi_{12,29}, \chi_{31,29}=\chi_{33,29}, \chi_{37,35}=\chi_{39,35}$, etc. For ANs of higher generation, the previous identical limiting probabilities are preserved and additional identical values of transition probabilities are observed due to structural symmetry of the network.

In summary, we have studied coherent exciton transport modeled by continuous-time quantum walks on ANs. The quantum transport exhibits a very distinct behavior compared to the classical random walks. For networks up to the second generation the coherent transport shows perfect recurrences when the initial excitation starts at the central node 7]. For networks of higher generation, the recurrence ceases to be perfect, which resembles results for discrete quantum carpets [7]. The excitation depends on the initial nodes and is most likely to be found at the original nodes while the coherent transport to other nodes is particularly low. In the long time limit, the transition probabilities show identical values between different nodes, which reflects the symmetry of the network structure.

We would like to point out that although CTQWs on ANs show oscillation and revivals like the results of the $1 \mathrm{D}$ case, there are some difference in the quantum dynamics between the two structures. For ANs, we find that the return probabilities at the central nodes are nearly periodic, in contrast to the $1 \mathrm{D}$ case where the (maximums of) return probability shows a power law decay as $\pi(t) \sim t^{-1}$ [21, 25]. In Ref. 25], the authors find that for a $1 \mathrm{D}$ chain, quantum revivals do not repeat indefinitely but become less and less accurate as time progresses [25]. They also find that the quantum walks displays Anderson localizations or decoherence in the presence static or dynamic disorder [25]. For ANs, there are also considerable localizations on the initial nodes (See Fig. 6). Such localizations may relate to the network structures and requires a further study.

This work is supported by National Natural Science Foundation of China under Project Nos 10575042, 10775058 and MOE of China under contract number IRT0624 (CCNU).

[1] J. Kempe, Contemp. Phys. 44, 307 (2002). 
[2] G. H. Weiss, Aspect and Applications of the Random Walk (North-Holland, Amsterdam, 1994).

[3] J.-P. Bouchaud and A. Georges, Phys. Rep. 195, 127 (1990).

[4] D. Solenov and L. Fedichkin, Phys. Rev. A 73, 012313(2003).

[5] H. Krovi and T. A. Brun, Phys. Rev. A 73, 032341 (2006).

[6] E. M. Bollt and D. ben-Avraham, New J. Phys. 726 (2005).

[7] O. Mülken, V. Bierbaum and A. Blumen, J. Chem. Phys 124, 124905 (2006).

[8] A. Blumen, V. Bierbaum and O. Mülken, Physica A 371, 10 (2006).

[9] N. Ashwin and V. Ashvin, quant-ph/0010117.

[10] O. Mülken and A. Blumen, Phys. Rev. E 71, 016101 (2005).

[11] O. Mülken, V. Pernice and A. Blumen, Phys. Rev. E 76, 051125 (2007).

[12] R. Albert and A.-L. Barabäsi, Rev. Mod. Phys 74, 47 (2002).

[13] S. Boccaletti, V. Latora, Y. Moreno, M. Chavez and D.U. Hwang, Phys. Rep. 424, 175 (2006).

[14] J.S. Andrade, H.J. Herrmann, R.F.S. Andrade, and
L.R.da Silva, Phys. Rev. Lett. 94, 018702 (2005).

[15] J. P. K. Doye and C. P. Massen, Phys. Rev. E 71, 016128 (2005).

[16] A. M. Souza and H. Herrmann, Phys. Rev. B 75, 054412 (2007).

[17] A. P. Vieira, J. S. Andrade, H. J. Herrmann and R. F. Andrade, Phys. Rev. E 76, 026111 (2007).

[18] P. G. Lind, J. A. C. Gallas and H. J. Herrmann, Phys. Rev. E 70, 056207 (2004).

[19] O. Mülken and A. Blumen, Phys. Rev. E 71, 016101 (2005).

[20] O. Mülken and A. Blumen, Phys. Rev. E 71, 036128 (2005).

[21] X. P. Xu, Phys. Rev. E 77, 061127 (2008).

[22] R.F.S. Andrade and J.G.V. Miranda, Physica A 356, 1 (2005).

[23] R. Iwanow, D. A. May-Arrioja, D. N. Christodoulides, G. I. Stegeman, Y. Min, and W. Sohler, Phys. Rev. Lett. 95, 053902 (2005).

[24] F. Grossmann, J.-M. Rost, and W. P. Schleich, J. Phys. A 30, L277 (1997).

[25] Y. Yin, D. E. Katsanos, and S. N. Evangelou, Phys. Rev. A 77, 022302 (2008). 